\title{
AVALIAÇÃO DA PRECIPITAÇÃO OCULTA NA SERRA DO MAR COM COLETORES PASSIVOS DE NEVOEIRO
}

\author{
ARCOVA, Francisco Carlos Soriano - arcova@if.sp.gov.br \\ Instituto Florestal de São Paulo \\ GALVANI, Emerson - egalvani@usp.br \\ Universidade de São Paulo / USP \\ RANZINI, Maurício - ranzini@if.sp.gov.br \\ Instituto Florestal de São Paulo \\ DE CICCO, Valdir - valdir.cicco@gmail.com \\ Instituto Florestal de São Paulo
}

\begin{abstract}
RESUMO: Nevoeiros de montanha são frequentes no Parque Estadual da Serra do Mar, em Cunha-SP, onde está localizado o Laboratório de Hidrologia Florestal Walter Emmerich. Nesse ambiente é importante avaliar a precipitação oculta com coletores de água de nevoeiro a fim de subsidiar as pesquisas em microbacias experimentais recobertas com Mata Atlântica. Entre outubro de 2009 e dezembro de 2012, foi desenvolvido estudo com esse objetivo. Realizaram-se coletas com dois coletores passivos cilíndricos - um coberto e outro descoberto - e um retangular descoberto, instalados na estação meteorológica do laboratório. Houve coleta de água ao longo dos três anos e os maiores volumes incidiram no outono e no inverno. As taxas de coleta foram reduzidas (média de $290,5 \mathrm{~mL} \cdot \mathrm{m}^{-2} \cdot \mathrm{d}^{-1}$ ). A curta duração dos episódios de nevoeiro de montanha, a baixa velocidade do vento no local e a ocorrência de eventos de nevoeiro de vale contribuíram para a pouca coleta de água.
\end{abstract}

PALAVRAS-CHAVES: nevoeiro de montanha, precipitação de nevoeiro, coleta de nevoeiro, neblina, floresta nebular.

\section{EVALUATION OF OCCULT PRECIPITATION IN THE SERRA DO MAR WITH PASSIVE FOG COLLECTORS}

ABSTRACT: Mountain fogs are frequent in the Serra do Mar State Park, in Cunha-São Paulo, Brazil, where Walter Emmerich Forest Hydrology Laboratory is located. In this environment it is important to evaluate the occult precipitation in fog water collectors in order to subsidize the research in experimental catchments. From October 2009 to December 2012, a study was carried out with this objective. Fog water was collected with two cylindrical passive collectors (one covered and one discovered) and one rectangular discovered, installed in the meteorological station of the laboratory. There was water collection over the three years and the largest volumes were in the fall and winter seasons. The collection rates were reduced (mean of $290,5 \mathrm{~mL} \cdot \mathrm{m}^{-2} \cdot \mathrm{d}^{-1}$ ). The short duration of the mountain fog episodes, the low speed of the winds and occurrence of valley fogs contributed to the little water collection.

KEYWORDS: mountain fog, fog precipitation, fog collection, mist, cloud forest.

\section{INTRODUÇÃO}

Os nevoeiros consistem em gotas de água de 1 a $40 \mu \mathrm{m}$ de diâmetro em suspensão na atmosfera e velocidade de queda inferior a $5 \mathrm{~cm} . \mathrm{s}^{-1}$. Mesmo em condições de ventos fracos eles se movimentam quase horizontalmente (WORLD METEOROLOGICAL ORGANIZATION, 2008).

Os nevoeiros podem ser classificados em sete tipos principais (BRUIJNZEEL; EUGSTER; BURKARD， 2005; EUGSTER，2008): nevoeiro de 
radiação - é o mais comum nas áreas interiores e incide em locais onde o ar frio tende a se acumular durante a noite ou no inverno. Para ele se desenvolver há necessidade de a atmosfera estar estável (ventos fracos), com uma camada de inversão térmica adjacente à superfície do solo. O processo que predomina na sua formação é o resfriamento da superfície em decorrência da perda radioativa da Terra, o qual reduz a temperatura abaixo do ponto de orvalho. O excesso de vapor de água é condensado nos aerossóis presentes; nevoeiro marítimo - é formado em áreas oceânicas abertas. Diferentes processos podem gerá-lo, mas o mais característico é o resfriamento do ar quente e úmido a uma temperatura inferior ao ponto de orvalho, quando o ar se desloca sobre a superfície da água. Sua ocorrência não se restringe à ventos fracos e pode se deslocar a grandes distâncias; nevoeiro de vapor - se forma sobre a superfície do mar, rios e lagos quando o ar frio com baixa capacidade de saturação de vapor de água flui sobre a superfície quente a uma taxa de evaporação da água maior do que o ar frio pode reter. O excesso de vapor é condensado em minúsculas gotas e gera uma névoa ascendente pouco espessa; nevoeiro de advecção - ocorre quando o ar quente e úmido se desloca horizontalmente sobre uma superfície fria, resfriando por contado e também por mistura com o ar frio até atingir a saturação. Em áreas costeiras, onde há forte contraste no aquecimento entre a terra e o oceano, pode ser gerada uma brisa marítima que acaba por empurrar o nevoeiro formado em alto-mar em direção ao continente; nevoeiro costeiro - é um típico nevoeiro de advecção que é formado quando a superfície terrestre é mais quente que a superfície fria do oceano. Os ventos transportam o nevoeiro denso que se forma no mar em direção à costa; nevoeiro de vale - é tipicamente um nevoeiro de radiação que se forma entre morros e montanhas. Ocorre normalmente no inverno, podendo perdurar por vários dias se o clima estiver estável, e nevoeiro de montanha - também chamado de nevoeiro orográfico ou de encosta, forma-se devido ao encontro do ar úmido com uma montanha, resultando no resfriamento do ar por expansão adiabática à medida que ele se move para maiores altitudes. É um dos poucos tipos de nevoeiro que se mantém em condições de vento forte, pois quanto maior a velocidade do vento mais veloz será a ascensão do ar e mais rápido será o seu resfriamento. Culmina em uma densa e extensa camada de nevoa sobre as encostas e montanhas, assemelhando-se a nuvens.

Os nevoeiros ganham importância hidrológica quando suas gotículas entram em contato com uma barreira física permeável, se aglutinam e formam gotas maiores que, pela ação da gravidade, se desprendem da superfície e se precipitam. Os nevoeiros podem ter a água captada por coletores construídos especificamente para este fim (SCHEMENAUER; CERECEDA, 1994a, b; FISCHER; STILL, 2007; ABDUL-WAHAB; LEA, 2008; HOLWERDA et al., 2010; FRUMAU et al., 2011; BAÊTA, 2012) e, também, naturalmente pela vegetação de grande porte, como árvores isoladas e florestas (BRUIJNZEEL; EUGSTER; BURKARD, 2005; MCJANNET et al., 2006; LIU et al., 2008; HOLWERDA; BRUIJNZEEL; SCATENA, 2010; PRADA et al., 2012). Esse processo é conhecido pelos hidrólogos como precipitação oculta, precipitação horizontal, precipitação de nevoeiro e precipitação por interceptação direta de nevoeiro (PRADA; SILVA, 2001).

Em comunidades de regiões áridas remotas a precipitação oculta captada em grandes coletores pode ser uma fonte alternativa de água para abastecimento humano, dessedentação de animais, irrigação de culturas 
agrícolas e de reflorestamentos (INTERNATIONAL DEVELOPMENT RESEARCH CENTRE, 1993; MARZOL, 2002; VALIENTE et al., 2010). Por sua vez, as copas das árvores são excepcionais coletoras naturais da água de nevoeiro, pois são permeáveis à passagem de ar, têm grande superfície de retenção e possibilitam o movimento contínuo das folhas e galhos pela ação do vento (PRADA et al., 2012). As florestas precipitam a água retida nas copas principalmente por gotejamento das folhas. Assim, para essas matas, a captação de água da atmosfera constitui-se em um processo do ciclo hidrológico e do balanço hídrico das bacias hidrográficas (BRUIJNZEEL; HAMILTON, 2001; MCJANNET et al., 2006). Os nevoeiros orográficos podem influenciar fortemente o balanço hídrico das florestas de regiões montanhosas (BRUIJNZEEL; EUGSTER; BURKARD, 2005).

Para a avaliação da viabilidade do uso de nevoeiros como fonte suplementar de água em regiões áridas e para localidades onde a captação de água ocorre naturalmente pela floresta, estudos são necessários para conhecer o potencial de coleta de água dos nevoeiros, a frequência de ocorrência dos mesmos, os aspectos relacionados à sazonalidade, dentre outros. Essas pesquisas são realizadas com coletores de água de nevoeiro (FRUMAU et al., 2011). Há uma série de coletores, em sua maioria de fabricação artesanal, que vem sendo utilizada para medição da precipitação oculta (BRUIJNZEEL; EUGSTER; BURKARD, 2005). O princípio de funcionamento dos mesmos é que ao passar por uma superfície vertical sólida e vazada as gotículas de água do nevoeiro impulsionadas pelo vento são depositadas por impacto, se aglutinam em gotas maiores e drenam para um reservatório posicionado logo abaixo da superfície coletora (OLIVIER, 2002; KLEMM et al., 2012, MONTECINOS; CERECEDA; RIVERA, 2018). Como funcionam exclusivamente pela ação do vento (não necessitam de uma fonte de energia para operar) são denominados de coletores "passivos" de nevoeiro (FISCHER; STILL, 2007).

Os coletores passivos de nevoeiro variam quanto ao material empregado na superfície coletora da água e na forma (desenho). Os tipos de superfície mais usados são a malha, confeccionada de linhas horizontais e verticais entrelaçadas e as linhas verticais paralelas, denominadas de "harpa" (FISCHER; STILL, 2007). A malha mais comum é a tela de sombreamento de polipropileno (sombrite), utilizada por Schemenauer e Cereceda (1994b) no coletor por eles idealizado como equipamento padrão nas pesquisas sobre coleta de água de nevoeiro. Pode ser considerado um material de referência na confecção de coletores, pois é amplamente empregado nos experimentos ao redor do mundo (PRADA; SILVA, 2001; OLIVIER; RAUTENBACH, 2002; MARZOL, 2002; OSSES; SCHEMENAUER; LEIBBRAND, 2004; MILETA, 2008; SCHEMENAUER; OSSES; LEIBBRAND, 2004; AL-HASSAN, 2009). Com relação à superfície do tipo "harpa", o fio de poliamida (linha de nylon) é o mais usado, como nos coletores construídos por Goodman (1985), Valiente, Estrela e Corell (2004), Fischer e Still (2007), Estrela et al. (2008), Mousavi-Baygi (2008), Frumau et al. (2010), Valiente et al.(2011), Baêta (2012). Quanto à forma são adotadas a plana e a cilíndrica. A primeira consiste em uma moldura retangular na qual é instalada a malha ou são passados verticalmente os fios de nylon. O coletor proposto por Schemenauer e Cereceda (1994b), batizado como SFC - standard fog collector é muito utilizado e consiste de uma estrutura de metal de $1 \mathrm{~m} \times 1 \mathrm{~m}$ com malha de polipropileno. Em pesquisas nas ilhas Canárias e no Marrocos, Marzol, Sanches e Yanes (2011) fizeram uso, além do SFC, de outros coletores com 1/4 
da sua dimensão, batizados como QFC - quarter size fog collectors. Goodman (1985) e Mousavi-baygi (2008) também usaram estrutura de ferro de $1 \mathrm{~m}^{2}$, porém com superfície coletora de linhas de nylon. Os coletores cilíndricos também são amplamente utilizados (GOODMAN, 1985; ESTRELA et al., 2008; MOUSAVI-BAYGI, 2008; VALIENTE et al., 2011; BAÊTA, 2012). As vantagens e desvantagens no uso de coletores cilíndricos e planos, bem como no material empregado na superfície coletora são discutidas por Fischer e Still (2007).

Um panorama geral das taxas diárias de precipitação oculta em coletores passivos ao redor do mundo é apresentado na Tabela 1. As taxas variam amplamente entre as regiões em decorrência de especificidades de cada sítio como a frequência com que os nevoeiros ocorrem ao longo do ano, a duração dos episódios, a velocidade do vento durante o evento, a distância em relação à costa e o tipo de nevoeiro, dentre outros. Sítios com alta incidência de nevoeiro ao longo do ano, normalmente, apresentam elevado potencial de coleta, como as Ilhas Canárias: 200 dias/ano (MARZOL; SANTOS, 2004) e Ilha da Madeira: 235 dias/ano (PRADA et al., 2004). O mesmo ocorre em relação à duração do nevoeiro (OLIVIER, 2002; BRUIJNZEEL; EUGSTER; BURKARD, 2005). Quanto maior a duração do episódio (maior tempo de imersão) maior é a taxa de água coletada (ESTRELA et al., 2008). A importância da velocidade do vento sobre a coleta de água de nevoeiro foi documentada em vários estudos (SCHEMENAUER; JOE; 1989; OLIVIER, 2002; MARZOL; SANTOS, 2004; ESTRELA et al., 2008; HIATT; FERNANDEZ; POTTER, 2012). Há uma tendência de aumento nas taxas de coleta com a velocidade do vento. Isso se deve ao fato de que ventos mais rápidos transportam mais gotas de nevoeiro horizontalmente para a superfície coletora (HIATT; FERNANDEZ; POTTER, 2012). Quanto à distância do sítio em relação à costa, há propensão de diminuição na incidência de nevoeiros à medida que se afasta do mar (CERECEDA et al., 2002). No entanto, variações causadas pela topografia regional podem levar à um desempenho oposto a essa tendência, com maiores coletas em sítios interiores (MARZOL; MEGIA, 2008). As taxas de coleta dependem, também, do tipo de nevoeiro. O orográfico e o advectivo, que normalmente incidem sob ventos mais intensos, são os que mais cedem água aos coletores. O de radiação, que ocorre sob ventos brandos, deposita escassas quantidades (BRUIJNZEEL; EUGSTER; BURKARD, 2005; ABDUL-WAHAB; LEAE, 2008).

Há uma considerável faixa tropical da Terra propícia à formação de nevoeiros. São localidades que sofrem influência do ar úmido proveniente dos oceanos. Essas áreas têm florestas naturais que funcionam como interceptadoras de água de nevoeiro. Normalmente, estes biomas situam-se em altitudes superiores a 1000 metros. Por se encontrarem frequentemente envoltos em nevoeiro recebem a denominação de "florestas tropicais nebulares montanas" (BRUIJNZEEL, 2001; BRUIJNZEEL; HAMILTON, 2001; BUBB et al., 2004). No Brasil, as florestas nebulares são constituídas pela Floresta Ombrófila Densa Montana e Floresta Ombrófila Densa Alto-Montana da Serra do Mar e do alto dos planaltos e das serras na Amazônia, como o Pico da Neblina e o Monte Roraima, e pela Floresta Ombrófila Mista na Serra da Mantiqueira (ARCOVA, 2013).

No Parque Estadual da Serra do Mar-PESM no município de Cunha-SP é comum a ocorrência de nevoeiros de montanha ao longo do ano. Nessa localidade está instalado o Laboratório de Hidrologia Florestal Walter Emmerich- 
LHFWE, onde pesquisas em microbacias recobertas com Mata Atlântica são realizadas desde 1980 . Quando da implementação do laboratório já se indagava sobre a possível influência da precipitação oculta na floresta para a entrada de água nas microbacias experimentais a serem implementadas e aventava-se a necessidade de realizar tais estudos no futuro. Essa demanda foi reiterada a medida que as investigações sobre o balanço hídrico foram aprofundadas (ARCOVA, 1996). No período de três anos, Arcova (2013) desenvolveu pesquisa com o escopo de obter informações sobre as potencialidades hidrológicas dos nevoeiros na região.

Nesse artigo são apresentados e discutidos os resultados da determinação da precipitação oculta em coletores passivos de água de nevoeiro instalados no posto meteorológico do LHFWE. 
Tabela 1 - Precipitação oculta média e máxima diária $\left(\mathrm{L} \cdot \mathrm{m}^{-2} \cdot \mathrm{d}^{-1}\right)$ em coletores passivos ao redor do mundo.

\begin{tabular}{|c|c|c|c|c|}
\hline Local & $\begin{array}{l}\text { Média diária } \\
\left(\mathrm{L} \cdot \mathrm{m}^{-2} \cdot \mathrm{d}^{-1}\right)\end{array}$ & $\begin{array}{l}\text { Máx. diária } \\
\left(\mathrm{L} \cdot \mathrm{m}^{-2} \cdot \mathrm{d}^{-1}\right)\end{array}$ & Período do estudo & Autor \\
\hline $\begin{array}{c}\text { Brand se Baai, África do } \\
\text { SuL }\end{array}$ & $0,36^{*}$ & 0,69 & Out. 1995 a Jun. 1997 & Olivier (2002) \\
\hline Guajira, Colombia & $1,41^{* *}$ & 3,25 & Jan. 2003 a Jan. 2004 & Garcia e Arango (2004) \\
\hline Anaga, I. Canárias & $11,5^{* * *}$ & - & 1996 a 2003, verão & Marzol e Garcia-Santos (2004) \\
\hline $\begin{array}{l}\text { C. Feiteiras, Ilha } \\
\text { Madeira }\end{array}$ & $2,9^{* * *}$ & - & 1996 a 2005 & Prada et al. (2004) \\
\hline Bica Cana, Ilha Madeira & $8,2^{* * *}$ & - & 1996 a 2005 & Prada et al. (2004) \\
\hline Boulaalam, Marrocos & $1,9^{* * *}$ & 66,0 & Jun. 2006 a nov. 2007 & Marzol e Megia (2008) \\
\hline C. Andes, Colombia & $3,3^{* * *}$ & - & 01 nov. 2003 a 01 fev. 2005 & Molina e Escobar (2008) \\
\hline Mt. Velebit, Croácia & - & 19,0 & 2000 a 2007, verão & Mileta (2008) \\
\hline Khorassan, Iran & $0,53^{* * *}$ & - & Mar. 2004 a Ago. 2005 & Mousavi-Baygi (2008) \\
\hline $\begin{array}{l}\text { Mt. Los Machos, } \\
\text { Espanha }\end{array}$ & $3,3^{*}$ & - & 2007 a 2009 & Valiente et al. (2011) \\
\hline $\begin{array}{l}\text { Boutmezguida, } \\
\text { Marrocos }\end{array}$ & $8,7^{* *}$ & - & Jun. 2006 a Set. 2010 & Marzol, Sanches e Yanes (2011) \\
\hline
\end{tabular}




\begin{tabular}{c|c|c|c|c}
\hline Ilhas Canárias, Espanha & $6,9^{* *}$ & - & Jun. 2006 a Set. 2010 & Marzol, Sanches e Yanes (2011) \\
\hline Big Sur-California, EUA & $2,3^{* * *}$ & 13,6 & 2010 e 2011, verão & Hiatt, Fernandez e Potter (2012) \\
\hline Big Sur-California, EUA & $3,9^{* * *}$ & 17,8 & 2010 e 2011, verão & Hiatt, Fernandez e Potter (2012) \\
\hline El Sarco, Chile & $2,9^{* *}$ & - & Jul. 2014 a Jun. 2015 & Montecinos, Cereceda e Rivera (2018) \\
\hline
\end{tabular}

* taxa abrangendo apenas os dias com coleta de água; ** taxa abrangendo todos os dias de coleta do estudo, isto é, dias com nevoeiro/chuva e dias secos (sem nevoeiro/chuva); *** não especificado no estudo se é taxa abrangendo apenas os dias com coleta de água ou taxa abrangendo todos os dias de coleta do estudo. 


\section{MATERIAL E MÉTODOS}

ÁREA EXPERIMENTAL

O LHFWE está localizado no núcleo Cunha do Parque Estadual da Serra do Mar, no município de Cunha-SP (230 13' e $23^{\circ} 16^{\prime} \mathrm{S} ; 45^{\circ} 02^{\prime}$ e $45^{\circ} 05^{\prime} \mathrm{W}$ ) a quinze quilômetros do litoral norte do Estado (Figura 1). A área faz parte da bacia hidrográfica do Rio Paraíba do Sul. O núcleo Cunha abrange, em nível regional, uma parte do alto ao médio Vale do Rio Paraibuna, pertencente ao Planalto de Paraitinga, no Planalto Atlântico. Na região há predominância de espigões com cimos ondulados e traçados sinuosos, com altitudes que ultrapassam os 1000 metros e $15^{\circ}$ de declividade, configurando o aspecto de "mares de morros" (FURIAN; PFEIFER, 1986). As classes de níveis de altitude no núcleo Cunha são distribuídas entre 1000 e 1228 m (FURIAN, 1987).

A temperatura média anual no núcleo Cunha é de $16,7{ }^{\circ} \mathrm{C}$ (CICCO, 2004). Os meses mais quentes são janeiro, fevereiro e março. A partir de abril há uma progressiva diminuição da temperatura média até julho, mês que, em geral, apresenta os menores valores que, eventualmente, podem ser negativos. A partir de agosto as temperaturas aumentam gradualmente até dezembro (ARMANI, 2004). A área apresenta elevada umidade relativa do ar ao longo do ano. Os maiores valores são registrados em janeiro, fevereiro e março e os menores em julho, agosto e setembro (ARMANI, 2004). A umidade relativa média mensal do ar nos meses úmidos está entre $80 \%$ e $85 \%$, sendo inferior a $80 \%$ no restante do ano. A precipitação pluviométrica média anual é de 2.015 $\mathrm{mm}$ (período de 1982 a 2012).

O núcleo Cunha está exposto a ação de nevoeiros de montanha que resultam do transporte do ar úmido do Oceano Atlântico em direção ao continente pelo processo de brisa marítima. Ao encontrar a escarpa da Serra do Mar o ar passa por convecção forçada se elevando e condensa em pequenas gotículas que se mantêm suspensas na atmosfera. Em decorrência destas condições os episódios de nevoeiros são frequentes e envolvem toda a área de estudo (ARCOVA, 2013). Na região há predomínio das massas tropicais. As massas polares são pouco frequentes e conduzem à existência de um período menos úmido. A menor penetração do ar polar reduz a quantidade das precipitações. A proximidade da Serra do Mar com o Oceano Atlântico é responsável pela pluviosidade no inverno. A influência orográfica é principalmente exercida sobre a Massa Tropical Atlântica que produz chuva na orla litorânea (MONTEIRO, 1973). 
Revista Brasileira de Climatologia

ISSN: 2237-8642 (Eletrônica)

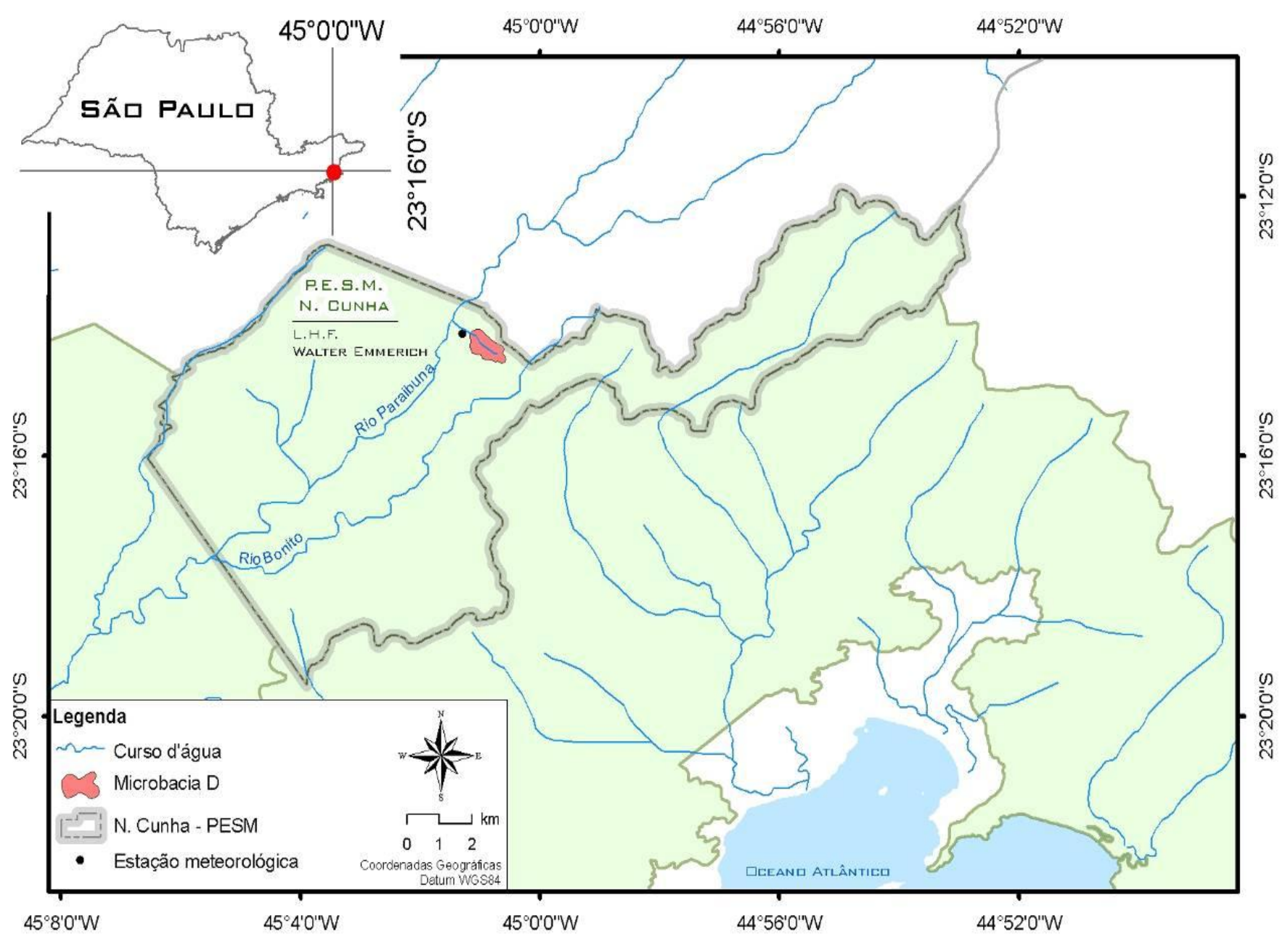

Figura 1 - Localização do PESM-Cunha/LHFWE (Fonte: Arcova, 2013). 
O núcleo Cunha está inserido no bioma Mata Atlântica. A vegetação é classificada como Floresta Ombrófila Densa, com formações Montana e AltoMontana (INSTITUTO BRASILEIRO DE GEOGRAFIA E ESTATÍSTICA, 2012). Ela é abundante em epífitas, com orquídeas e bromélias habitando os ramos e caule das árvores (ARCOVA, 2013).

\section{COLETORES PASSIVOS DE ÁGUA DE NEVOEIRO}

No estudo foram usados coletores passivos tipo "harpa": um retangular e dois cilíndricos (Figura 2).

O coletor retangular consiste de uma estrutura de ferro de área de captação de nevoeiro de $1 \mathrm{~m} \times 1 \mathrm{~m}$, cujas partes superior e inferior foram construídas com barra cilíndrica maciça de $1 / 2$ polegada de diâmetro e 1,30 m de comprimento, onde foram soldadas barras roscadas de $5 / 16$ de polegada de diâmetro e um metro de comprimento. As laterais foram confeccionadas em estrutura tubular de $2 \mathrm{~cm} \times 3 \mathrm{~cm}$ e 2,40 m de comprimento. As ranhuras das barras roscadas foram usadas para a passagem da linha de nylon de 0,80 mm de espessura, formando um painel de duas superfícies paralelas. Sob a superfície coletora foi instalada uma calha metálica e a ela acoplado um pluviógrafo (Davis Instruments, 0,2 mm/báscula) conectado a um sistema de aquisição de dados automático (Hobo) para registrar o volume de água. Um funil plástico com a "boca" virada para baixo foi instalado sobre a abertura de coleta do pluviógrafo para impedir a entrada de água da chuva. O conjunto foi fixado por parafusos e porcas a dois caibros de madeira, através de furos feitos nas estruturas tubulares.

Os coletores cilíndricos foram construídos com dois anéis de barra roscada soldados a quatro barras verticais de $0,6 \mathrm{~cm}$ de espessura. Assim como para o coletor retangular as ranhuras das barras roscadas foram usadas para a passagem da linha de nylon, formando um painel de duas superfícies de coleta. Um dos coletores tinha anéis de $26 \mathrm{~cm}$ de diâmetro e as barras de $46 \mathrm{~cm}$ de comprimento. A estrutura foi acoplada a um funil com $29 \mathrm{~cm}$ de diâmetro para drenar a água coletada para uma garrafa plástica de um litro. Foi coberto com uma chapa de compensado naval de $1,6 \mathrm{~m} \times 1,6 \mathrm{~m}$ suspensa por quatro caibros de madeira para evitar a entrada de água das chuvas. O outro coletor, com anéis de $23 \mathrm{~cm}$ de diâmetro e $43 \mathrm{~cm}$ de altura, foi montado sobre um pluviômetro e mantido exposto às chuvas.

Os três coletores foram instalados no posto meteorológico do LHFWE (1050 m de altitude) entre 2 e 2,5 $\mathrm{m}$ do solo. Mais detalhes dos coletores estão em Arcova e Galvani (2014). 


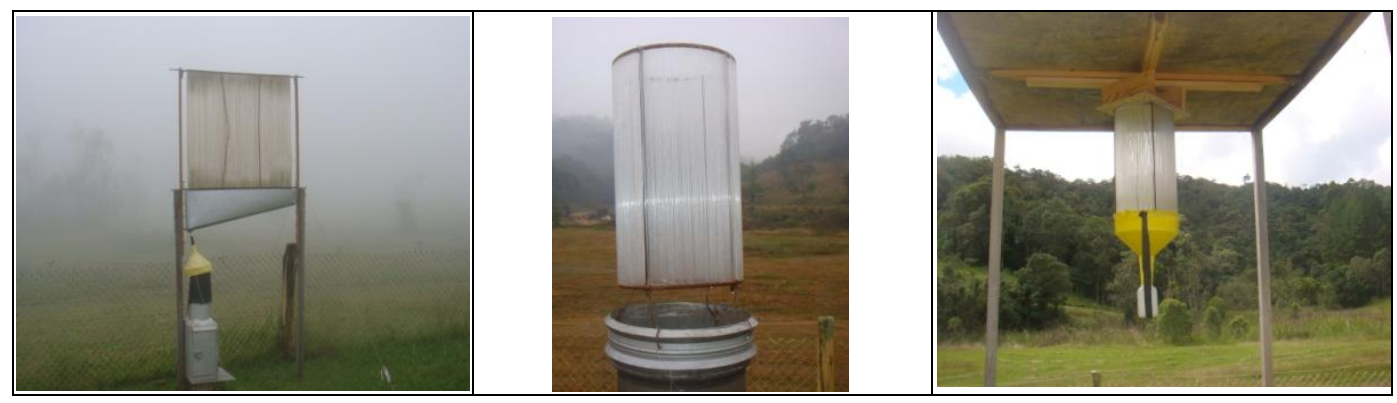

Figura 2 - Coletores de água de nevoeiro no posto meteorológico do LHFWE.

\section{PROCEDIMENTOS}

As coletas com o coletor cilíndrico coberto foram feitas de outubro de 2009 a setembro de 2012 e incluíram tanto eventos de nevoeiro com ocorrência de chuva como sem ela. As coletas realizadas com o coletor cilíndrico descoberto tiveram início em maio de 2010 e se estenderam até setembro de 2012. Os registros do pluviógrafo acoplado ao coletor plano foram usados para verificar a ocorrência de episódios de nevoeiro e o horário do início e do fim da coleta de água proporcionada em cada evento. Devido a problemas de funcionamento desse equipamento houve descontinuidade na coleta de dados. Os primeiros registros foram feitos entre outubro e novembro de 2010 e os demais entre setembro e novembro de 2012. Para os dois coletores descobertos foram selecionados somente os eventos nos quais não ocorreu chuva. Os registros do pluviógrafo do posto meteorológico do LHFWE (Nakaasa, 0,5 $\mathrm{mm} /$ báscula) foram analisados para averiguar quando choveu ou não. Considerou-se que a água retida nos coletores durante os eventos em que não houve registro no pluviógrafo originou-se unicamente de nevoeiro.

As coletas foram realizadas de segunda a sexta-feira, entre $08 \mathrm{~h} 00 \mathrm{~min}$ e 09h30min. A água coletada nos fins de semana foi medida na segunda-feira seguinte. Nas ocasiões em que as chuvas se prolongaram por mais de um dia as coletas foram efetuadas na manhã seguinte ao fim das mesmas. Os volumes por unidade de área $\left(\mathrm{mL} \cdot \mathrm{m}^{-2}\right.$ ) foram obtidos pela divisão das quantidades de água recolhidas e a área da seção transversal de cada coletor.

\section{RESULTADOS}

As medições no coletor cilíndrico coberto estenderam-se por 36 meses (Figura 3) e resultaram em 344 coletas (30\% do tempo amostrado). Alguns eventos se destacaram ao longo dos três anos com taxas superiores às demais. Os maiores valores no período de outubro de 2010 a setembro de 2011 se originaram do acúmulo de água de mais de um episódio de nevoeiro. As coletas dos dias 01 de novembro e 13 de dezembro de 2010 iniciaram na sexta-feira e se prolongaram até a manhã da segunda-feira. Os volumes recolhidos em 28 de junho e 01 de agosto de 2011 resultaram de coletas que perduraram por seis e cinco dias, respectivamente. Nos dois últimos eventos houve possibilidade de ter ocorrido mistura de água de nevoeiro com a de chuva e de garoa dirigidas pelos ventos. Nesses períodos houve registros característicos desses tipos de precipitação no pluviógrafo do posto meteorológico, isto é, pulsos de $0,5 \mathrm{~mm}$ 
separados por amplos intervalos de tempo. O evento mais expressivo do período de outubro de 2011 a setembro de 2012 ocorreu no dia 27 do último mês do estudo e totalizou $1418 \mathrm{~mL} \cdot \mathrm{m}^{-2}$. Este episódio se caracterizou pela ocorrência de chuva dirigida pelo vento, que persistiu das 7 às 17 horas do dia 26, acompanhada de rajadas de ventos de até $8,5 \mathrm{~m} \cdot \mathrm{s}^{-1}$, conforme registros anemométricos no posto meteorológico. Uma segunda chuva dirigida pelo vento que teve início nas primeiras horas do dia 27 pode ter contribuído para o montante de água interceptada pelo coletor.

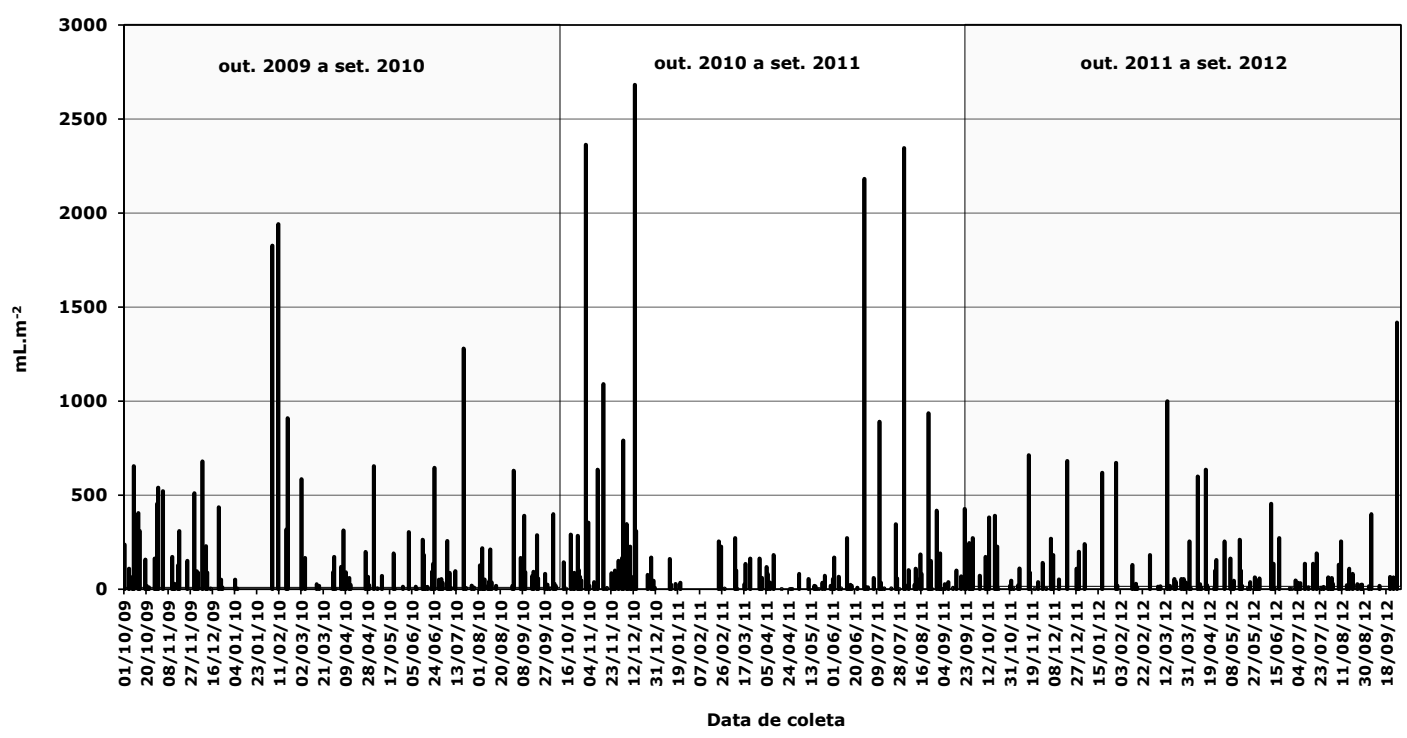

Figura 3 - Volumes de água de nevoeiro captados no coletor cilíndrico coberto.

Entre maio de 2010 e outubro de 2012, foram feitas 194 coletas de água de nevoeiro sem a presença de chuva no coletor cilíndrico descoberto (Figura 4). Os volumes variaram de 2,0 a $1.990 \mathrm{~mL} \cdot \mathrm{m}^{-2}$. Os montantes mais expressivos derivaram de eventos de fim de semana que podem ser resultado do acúmulo de água de até três dias (sexta-feira após a coleta, sábado, domingo e segundafeira de manhã). A taxa média diária, considerando apenas os dias com ocorrência de nevoeiro, foi calculada em $290,5 \mathrm{~mL} \cdot \mathrm{m}^{-2} \cdot \mathrm{d}^{-1}\left(0,290 \mathrm{~L} \cdot \mathrm{m}^{-2} \cdot \mathrm{d}^{-1}\right)$. As maiores coletas incidiram no outono e inverno (Figura 4). Em 2010, 2011 e 2012 as quantidades de água captadas nessas estações do ano totalizaram $14.226 \mathrm{~mL} . \mathrm{m}^{-2}, 21.298 \mathrm{~mL} \cdot \mathrm{m}^{-2}$ e $28.671 \mathrm{~mL} \cdot \mathrm{m}^{-2}$, respectivamente, contra 3.817 $\mathrm{mL} . \mathrm{m}^{2}$ e $6.464 \mathrm{~mL} \cdot \mathrm{m}^{-2}$ na primavera e verão. O número de eventos com nevoeiro também variou com a época do ano; foram 37, 49 e 58 no outono e inverno, e apenas 21 e 38 na primavera e verão. 


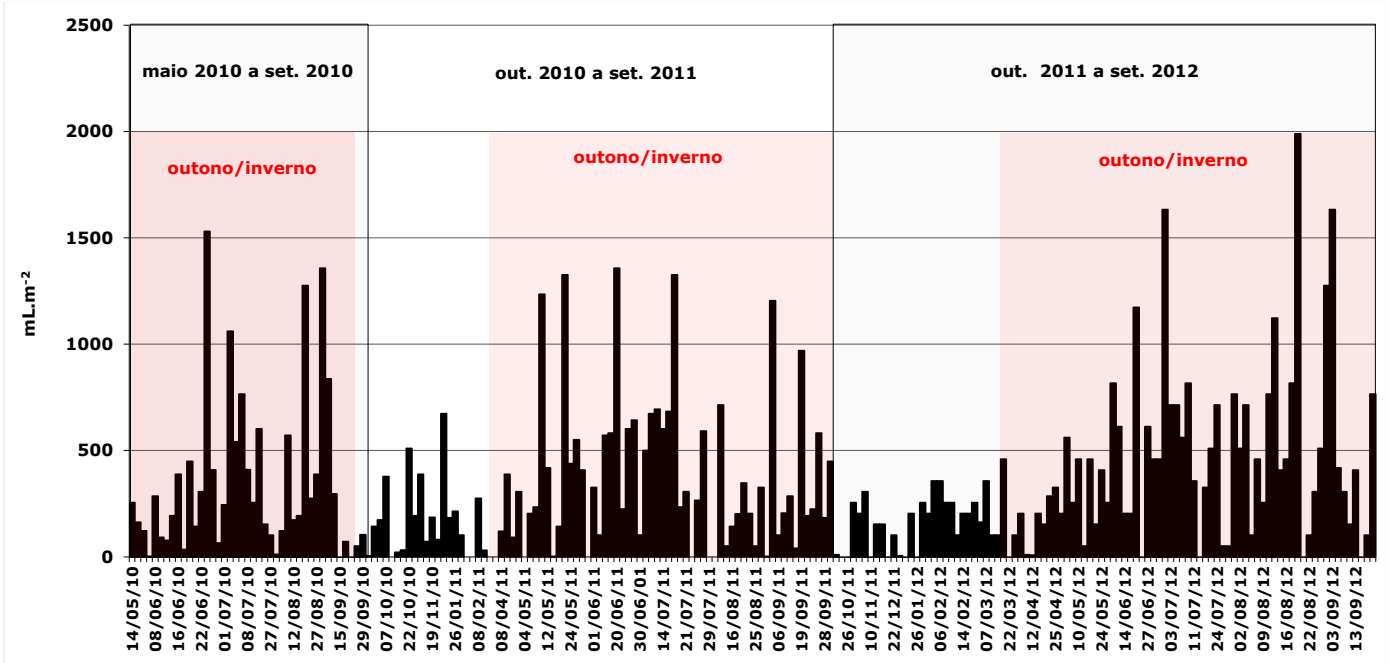

Data de coleta

Figura 4 - Volume de água de nevoeiro captado pelo coletor cilíndrico descoberto nos eventos sem chuva.

A Tabela 2, elaborada a partir dos dados do pluviógrafo acoplado ao coletor plano, mostra o horário do início, do fim e da duração dos registros de coleta de água ocasionados por eventos de nevoeiro sem a presença de chuva. Foram observados dois padrões distintos: registros de não mais que três horas de duração, que ocorreram a tarde, quando predominam os nevoeiros de montanha na região e outros que se prolongaram por várias horas, durante a noite, com muitos deles se estendendo até o alvorecer. Esses registros são oriundos de nevoeiros de vale. A formação desses tipos de nevoeiro na condição acima descrita foi observada várias vezes.

Tabela 2 - Informações obtidas dos registros do pluviógrafo acoplado ao coletor retangular de água de nevoeiro.

\begin{tabular}{|c|c|c|c|c|c|c|}
\hline \multirow{2}{*}{\multicolumn{2}{|c|}{ Evento }} & \multicolumn{2}{|c|}{$\begin{array}{c}\text { Horário (hora e minutos) } \\
\text { e dia do registro do } \\
\text { nevoeiro }\end{array}$} & \multirow[t]{2}{*}{$\begin{array}{l}\text { Duração do } \\
\text { registro }\end{array}$} & \multirow[t]{2}{*}{$\begin{array}{l}\text { Volume de } \\
\text { água (mL) }\end{array}$} & \multirow[t]{2}{*}{$\begin{array}{c}\text { Tipo de } \\
\text { nevoeiro }\end{array}$} \\
\hline & & Início & Fim & & & \\
\hline $\begin{array}{l}28 / 08 \\
29 / 08 / 10\end{array}$ & a & $\begin{array}{c}01 \text { h } 23 \\
\min 29 / 08\end{array}$ & $\begin{array}{c}08 \text { h } 17 \\
\min 29 / 08\end{array}$ & $\begin{array}{c}06 \mathrm{~h} 54 \\
\min \end{array}$ & 115,0 & Vale \\
\hline \multirow{2}{*}{\multicolumn{2}{|c|}{$\begin{array}{l}03 / 11 \\
04 / 11 / 10\end{array}$}} & $\begin{array}{c}15 \mathrm{~h} 12 \\
\min \end{array}$ & $\begin{array}{c}16 \mathrm{~h} 05 \\
\min \end{array}$ & $\begin{array}{c}00 \mathrm{~h} 53 \\
\min \end{array}$ & 75,0 & Montanha \\
\hline & a & $\begin{array}{c}22 \mathrm{~h} 42 \\
\min \end{array}$ & $\begin{array}{c}07 \mathrm{~h} 15 \\
\min \end{array}$ & $\begin{array}{c}08 \mathrm{~h} 33 \\
\min \end{array}$ & 195,0 & Vale \\
\hline $\begin{array}{l}04 / 11 \\
05 / 11 / 10\end{array}$ & a & $\begin{array}{c}23 \text { h } 45 \\
\min 04 / 11\end{array}$ & $\begin{array}{c}03 \text { h } 14 \\
\min 05 / 11\end{array}$ & $\begin{array}{c}02 \mathrm{~h} 29 \\
\min \end{array}$ & 95,0 & Vale \\
\hline \multirow{2}{*}{\multicolumn{2}{|c|}{$\begin{array}{l}18 / 11 \\
19 / 11 / 10\end{array}$}} & $\begin{array}{c}15 \text { h } 24 \\
\min 18 / 11\end{array}$ & $\begin{array}{c}18 \text { h } 36 \\
\min 18 / 11\end{array}$ & $\begin{array}{c}03 \mathrm{~h} 00 \\
\min \end{array}$ & 65,0 & Montanha \\
\hline & & $\begin{array}{r}23 \text { h } 55 \\
\min 18 / 11 \\
\end{array}$ & $\begin{array}{c}06 \text { h } 11 \\
\min 19 / 11\end{array}$ & $\begin{array}{c}05 \mathrm{~h} 16 \\
\min \end{array}$ & 115,0 & Vale \\
\hline
\end{tabular}




\begin{tabular}{|c|c|c|c|c|c|}
\hline $\begin{array}{l}29 / 11 \\
30 / 11 / 10\end{array}$ & $\begin{array}{c}02 \mathrm{~h} 17 \\
\min 30 / 11\end{array}$ & $\begin{array}{c}02 \mathrm{~h} 42 \\
\min 30 / 11\end{array}$ & $\begin{array}{c}00 \mathrm{~h} 25 \\
\min \end{array}$ & 15,0 & Vale \\
\hline $\begin{array}{l}18 / 09 / 12 \text { a } \\
19 / 09 / 12\end{array}$ & $\begin{array}{r}22 \text { h } 30 \\
\min 18 / 09\end{array}$ & $\begin{array}{c}01 \text { h } 10 \\
\min 19 / 09\end{array}$ & $\begin{array}{c}02 \mathrm{~h} 40 \\
\min \end{array}$ & 40,0 & Vale \\
\hline $\begin{array}{l}01 / 10 / 12 a \\
02 / 10 / 12\end{array}$ & $\begin{array}{c}21 \text { h } 50 \\
\min 01 / 10\end{array}$ & $\begin{array}{c}07 \text { h } 21 \\
\min 02 / 10\end{array}$ & $\begin{array}{c}09 \mathrm{~h} 31 \\
\min \end{array}$ & 55,0 & Vale \\
\hline $\begin{array}{l}02 / 10 / 12 \text { a } \\
03 / 10 / 12\end{array}$ & $\begin{array}{c}22 \mathrm{~h} 42 \\
\min 02 / 10\end{array}$ & $\begin{array}{c}06 \text { h } 12 \\
\min 03 / 10\end{array}$ & $\begin{array}{c}07 \mathrm{~h} 30 \\
\min \end{array}$ & 150,0 & Vale \\
\hline $\begin{array}{l}03 / 10 / 12 a \\
04 / 10 / 12\end{array}$ & $\begin{array}{c}22 \mathrm{~h} 16 \\
\min 03 / 10\end{array}$ & $\begin{array}{c}06 \text { h } 33 \\
\min 04 / 10\end{array}$ & $\begin{array}{c}08 \mathrm{~h} 17 \\
\min \end{array}$ & 140,0 & Vale \\
\hline $\begin{array}{l}08 / 10 / 12 \text { a } \\
09 / 10 / 12\end{array}$ & $\begin{array}{c}20 \text { h } 52 \\
\min 08 / 10\end{array}$ & $\begin{array}{c}00 \mathrm{~h} 56 \\
\min 09 / 10\end{array}$ & $\begin{array}{c}04 \mathrm{~h} 04 \\
\min \end{array}$ & 75,0 & Vale \\
\hline $\begin{array}{l}09 / 10 / 12 \text { a } \\
10 / 10 / 12\end{array}$ & $\begin{array}{c}21 \mathrm{~h} 01 \\
\min 09 / 10\end{array}$ & $\begin{array}{c}21 \mathrm{~h} 49 \\
\min 09 / 10\end{array}$ & $\begin{array}{c}00 \mathrm{~h} 48 \\
\min \end{array}$ & 15,0 & Vale \\
\hline $\begin{array}{l}15 / 10 / 12 \text { a } \\
16 / 10 / 12\end{array}$ & $\begin{array}{c}00 \mathrm{~h} 43 \\
\min 16 / 10\end{array}$ & $\begin{array}{c}06 \text { h } 17 \\
\min 16 / 10\end{array}$ & $\begin{array}{c}05 \mathrm{~h} 34 \\
\min \end{array}$ & 85,0 & Vale \\
\hline $\begin{array}{l}16 / 10 / 12 \mathrm{a} \\
17 / 10 / 12\end{array}$ & $\begin{array}{c}22 \mathrm{~h} 36 \\
\min 16 / 10 \\
\end{array}$ & $\begin{array}{c}04 \mathrm{~h} 19 \\
\min 17 / 10 \\
\end{array}$ & $\begin{array}{c}05 \mathrm{~h} 43 \\
\min \end{array}$ & 75,0 & Vale \\
\hline \multirow{2}{*}{$\begin{array}{l}07 / 11 / 12 a \\
08 / 11 / 12\end{array}$} & $\begin{array}{c}13 \text { h } 27 \\
\min 07 / 11 \\
\end{array}$ & $\begin{array}{c}16 \mathrm{~h} 05 \\
\min 08 / 11 \\
\end{array}$ & $\begin{array}{c}02 \mathrm{~h} 38 \\
\min \end{array}$ & 150,0 & Montanha \\
\hline & $\begin{array}{c}01 \text { h } 58 \\
\min 08 / 11\end{array}$ & $\begin{array}{c}07 \text { h } 20 \\
\min 08 / 11\end{array}$ & $\begin{array}{c}05 \mathrm{~h} 18 \\
\min \end{array}$ & 60,0 & Vale \\
\hline $\begin{array}{l}21 / 11 / 12 \text { a } \\
22 / 11 / 12\end{array}$ & $\begin{array}{l}21 \mathrm{~h} 33 \\
\min 21 / 11\end{array}$ & $\begin{array}{llr}06 & h & 57 \\
\min & 22 / 11 \\
\end{array}$ & $\begin{array}{lll}09 & h & 24 \\
\min & & \\
\end{array}$ & 65,0 & Vale \\
\hline \multirow{2}{*}{$\begin{array}{l}22 / 11 / 12 a \\
23 / 11 / 12\end{array}$} & $\begin{array}{c}15 \text { h } 31 \\
\min 22 / 11 \\
\end{array}$ & 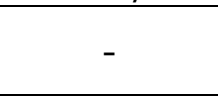 & $\begin{array}{c}02 \mathrm{~h} 50 \\
\min ^{*}\end{array}$ & 5,0 & Montanha \\
\hline & $\begin{array}{c}23 \text { h } 19 \\
\min 22 / 11\end{array}$ & $\begin{array}{c}08 \text { h } 15 \\
\min 23 / 11\end{array}$ & $\begin{array}{c}09 \mathrm{~h} 00 \\
\min \end{array}$ & 205,0 & Vale \\
\hline
\end{tabular}

* Duração do nevoeiro obtida de observação visual feita no campo.

\section{DISCUSSÃO}

A frequência de coleta de água de nevoeiro com o coletor coberto (Figura 3) foi inferior à registrada nas Ilhas Canárias, na qual de janeiro de 2000 a março de 2001 houve coleta em 325 dias (MARZOL, 2002), à da Ilha da Madeira onde houve 170 dias de coleta ao ano (PRADA et al., 2004) e também à de Porto Rico, onde houve coleta em 99 de 100 dias amostrados (HOLWERDA et al., 2010).

Os volumes variaram de 1,8 a $2.682 \mathrm{~mL} \cdot \mathrm{m}^{-2}$. A taxa média diária foi calculada em $77,9 \mathrm{~mL} \cdot \mathrm{m}^{2} \cdot \mathrm{d}^{-1}\left(0,08 \mathrm{~L} \cdot \mathrm{m}^{2} \cdot \mathrm{d}^{-1}\right)$. A cobertura instalada sobre 0 coletor cilíndrico foi eficiente na obstrução das chuvas verticais e pouco inclinadas, fato que foi observado no campo. Porém, a presença da placa de compensado e da estrutura que a suportava comprometeu as taxas de coleta da água de nevoeiro. Embora seja um artificio muito usado (JUVIK; NULLET, 1995; BRUIJNZEEL; EUGSTER; BURKARD，2005; HAFKENSCHEID, 2000; MCJANNET; WALLACE, 2006; HOLWERDA et al., 2010), a cobertura provoca alterações nos fluxos de vento ao redor do coletor, influenciando a captação de água (SCHEMENAUER; CERECEDA, 1995). 
A cobertura instalada sobre o coletor cilíndrico nem sempre foi capaz de evitar a mistura da água de nevoeiro com a de chuva. Em Cunha são frequentes os episódios de chuva de baixa intensidade e de garoa, que são de longa duração e caracterizadas por gotas de tamanho reduzido e que se movimentam quase horizontalmente. Esses eventos, normalmente, acontecem combinados com os nevoeiros. Assim, deve ser levado em conta que nestas ocasiões, à exemplo do que ocorreu nos dias 28/junho/2011, 01/agosto/2011 e $27 /$ setembro/2012 (Figura 3) as coletas podem ter resultado da mistura da água proveniente dos dois processos meteorológicos, indo ao encontro das dificuldades previstas por Bruijnzeel (2001), Bruijnzeel, Eugster e Burkard (2005), McJannet e Wallace (2006) e Frumau et al. (2011) para a quantificação exclusiva da água dos nevoeiros pelos coletores passivos. Em um estudo desenvolvido na Costa Rica a maior parte da água teve como origem as chuvas horizontais dirigidas pelo vento (FRUMAL et al., 2011). No Equador, a água coletada em cinco pontos de uma vertente também teve contribuição expressiva da chuva/garoa horizontal (BENDIX; FABIAN; ROLLENBECK, 2004).

A taxa média diária de precipitação oculta no coletor cilíndrico descoberto, considerando apenas os dias sem ocorrência de chuva (Figura 4), foi calculada em $290,5 \mathrm{~mL} \cdot \mathrm{m}^{-2} \cdot \mathrm{d}^{-1}\left(0,290 \mathrm{~L} \cdot \mathrm{m}^{-2} \cdot \mathrm{d}^{-1}\right)$. É uma taxa baixa quando comparada com a maior parte dos resultados de pesquisas desenvolvidas em várias partes do mundo (Tabela 1 ). Por exemplo, nas localidades de Feiteiras e Bica da Cana na Ilha da Madeira as taxas médias diárias, levando em conta somente os dias de nevoeiro (sem ocorrência de chuva), foram $2,9 \mathrm{~L} \cdot \mathrm{m}^{-2} \cdot \mathrm{d}^{-1} \mathrm{e}$ $8,9 \mathrm{~L} \cdot \mathrm{m}^{-2} \cdot \mathrm{d}^{-1}$ (PRADA et al., 2004). A taxa máxima diária de precipitação oculta obtida em Cunha também é baixa quando confrontada com a de sítios com forte potencial hídrico dos nevoeiros. Na Croácia foram coletados até $19 \mathrm{~L} . \mathrm{m}^{-2}$ em um único dia (MILETA, 2008) e no Marrocos 66 L.m ${ }^{-2} \cdot \mathrm{d}^{-1}$ (MARZOL; SÁNCHES; MEGIA, 2008).

Os totais de coleta superiores no outono e inverno (Figura 4) podem estar associados à dinâmica de circulação regional. Nessa latitude ocorre predomínio do Anticiclone no Atlântico Sul dinamizado pela Massa Tropical Atlântica. Esse padrão de circulação confere direções predominantes do quadrante leste, resultando em deslocamento de umidade do Oceano Atlântico em direção ao continente. Mesmo o Anticiclone consistindo-se em um centro de alta pressão (tempo estável), a parcela de ar úmido é forçada, mecanicamente, a se elevar no encontro com a Serra do Mar, resultando em resfriamento adiabático. Como a área de estudo está a $15 \mathrm{~km}$ do Oceano, essa influência é perceptível, em especial, nos meses de outono e inverno (MONTEIRO, 1973). Armani e Galvani (2011) estudando o setor nordeste do estado de São Paulo entre o inverno de 2008 ao outono de 2009 observaram que:

"Os resultados revelaram que houve 45 passagens frontais com duração mais frequente de um dia, controlando $21,9 \%$ do ano. Os intervalos mais frequentes, entre uma frente e outra, foram de 5 a 6 dias, oscilando entre um mínimo de 0 (com duas frentes num mesmo dia) a 18 dias. Os sistemas extratropicais controlaram a maior parte do tempo $(64,9 \%$ do ano), sendo que os anticiclônicos atuaram durante $43 \%$ do ano e os frontais, $21,9 \%$. O restante do tempo foi dominado pelos sistemas tropicais (Atlântico e Continental) $(35,1 \%)$, sendo que, desses, 
o sistema Atlântico atuou por $31,8 \%$ e o Continental, 3,3\%" (Op. Cit, p.7)

A exemplo de Cunha, variações sazonais da precipitação oculta foram observadas em vários estudos. Marzol (2002) observou que agosto é menos eficiente para obter água dos nevoeiros no Parque Rural Teno e Abril o mês mais propício. Na Namíbia, resultados de coleta de água apontaram o período de agosto a fevereiro como sendo o mais produtivo (SHANYENGANA et al., 2002). Na região de Tarapacá - Chile, o inverno e a primavera são as estações mais profícuas, com as maiores taxas de coleta ocorrendo de julho a setembro. Verão e outono incluem os meses com menores valores, provocando o decréscimo da taxa anual média de coleta (CERECEDA et al., 2002). Na costa atlântica do Marrocos as taxas são maiores no inverno e em Anaga - Ilhas Canárias no verão (MARZOL; SÁNCHES; YANES, 2011).

A despeito da grande frequência dos nevoeiros de vale no LHFWE, os volumes de água captados foram reduzidos, mesmo os eventos sendo de longa duração (Tabela 2). Esse tipo de nevoeiro ocorre sob ventilação branda o que restringe as taxas de coleta (CERECEDA et al., 2002; PRADA et al., 2004). Quanto aos nevoeiros orográficos, que também apresentaram taxas escassas de coleta (Tabela 2), ao menos dois fatores devem ter contribuindo para os resultados encontrados: a curta duração dos episódios de nevoeiro e a baixa velocidade do vento quando os mesmos incidiram. Apurou-se da rotina de observações de campo feitas no LHFWE que os nevoeiros orográficos tendem a se dissipar rapidamente. Os eventos que se prolongam por horas são pouco comuns. Os registros dos nevoeiros de montanha apresentados na Tabela 2 foram curtos e variaram de poucos minutos a não mais do que três horas. Eles são pouco expressivos quando comparados aos assinalados em regiões onde esse fenômeno assume importância hidrológica. Registros feitos nas Ilhas Canárias tiveram entre duas e cinco horas consecutivas de coleta. Porém, episódios mais duradouros foram frequentes, com dez a vinte horas seguidas, sendo o mais longo de sessenta e oito horas (MARZOL, 2002). Na África do Sul, aproximadamente $40 \%$ dos eventos duraram entre dez e vinte e cinco horas (LOUW; VAN HEERDEN; OLIVIER, 1998). Em Porto Rico, a duração foi de dezesseis horas em média (HOLWERDA et al., 2010).

Os coletores passivos necessitam de vento para captar a água de nevoeiro. Embora sob baixa ventilação possa haver coleta as taxas são menores do que as obtidas sob ventos mais intensos (SCHEMENAUER; JOE, 1989). Foi verificado que no período da tarde/início da noite de dias em que houve coleta de água pelo coletor coberto a velocidade média do vento no posto meteorológico do LHFWE foi de $1,4 \mathrm{~m} \cdot \mathrm{s}^{-1}$ (ARCOVA, 2013). Os ventos com velocidade igual ou inferior a $1,9 \mathrm{~m} . \mathrm{s}^{-1}$ prevaleceram sobre os demais, correspondendo a $74 \%$ do tempo. Assim no interstício das 12 as 20 horas, quando a ocorrência dos nevoeiros orográficos na área de estudo é mais provável, a intensidade dos ventos esteve em uma condição de baixa eficiência de coleta de água (SCHEMENAUER; JOE, 1989). 


\section{CONCLUSÕES}

Após três anos de avaliação da precipitação oculta em coletores passivos de nevoeiro no Parque Estadual da Serra do Mar em Cunha-SP, os resultados permitiram concluir:

- há coleta de água de nevoeiro ao longo das quatro estações do ano, com os maiores volumes incidindo no outono e inverno;

- as quantidades de água de nevoeiro coletadas em Cunha são consideradas baixas em comparação com as de estudos semelhantes realizados em outras partes do mundo;

- os escassos volumes de água retidos nos coletores resultam da combinação da ocorrência de nevoeiros de vale, a curta duração dos episódios dos nevoeiros de montanha associada a baixa intensidade dos ventos na região.

\section{REFERÊNCIAS BIBLIOGRÁFICAS}

ABDUL-WAHAB; S. A.; LEAE, V. Reviewing fog water collection worldwide and in Oman. International Journal of Environmental Studies, London, v. 65, n. 3, p. 487-500, 2008.

AL-HASSAN, G. A. Fog water collection evaluation in Asir Region-Saudi Arabia. Water Resources Management, Athens, v. 23, n. 13, p. 2805-2813, Oct. 2009.

ARCOVA, F. C. S. Balanço hídrico, características do deflúvio e calibragem de duas microbacias hidrográficas na Serra do Mar, SP. 1996. 155 f. Dissertação (Mestrado em Recursos Florestais) - Escola Superior de Agricultura "Luiz de Queiróz", Universidade de São Paulo, Piracicaba, 1996.

ARCOVA, F. C. S. Avaliação do potencial hidrológico dos nevoeiros e da precipitação oculta em ambiente de Floresta Ombrófila Densa Montana na Serra do Mar, Cunha, SP. 2013. 175 f. Tese (Doutorado em Geografia Física) Faculdade de Filosofia, Letras e Ciências Humanas, Universidade de São Paulo, São Paulo, 2013.

ARCOVA, F. C. S.; GALVANI, E. Instrumental para coleta de água de nevoeiro desenvolvido no Laboratório de Hidrologia Florestal Walter Emmerich, Cunha/SP-Brasil. In: SILVA, C. A.; FIALHO, E. S.; STEINKE (Org.). Experimentos em Climatologia Geográfica. Dourados, MS: UFGD, 2014. Cap. 16, p. 307-323.

ARMANI, G. Interações entre a atmosfera e a superfície terrestre: variações da temperatura e umidade na bacia B do Núcleo Cunha (IF) - SP. 2004. 198 f. Dissertação (Mestrado em Geografia Física) - Faculdade de Filosofia, Letras e Ciências Humanas, Universidade de São Paulo, 2004.

ARMANI, G.; GALVANI, E. Fluxos polares e o ritmo dos sistemas atmosféricos no nordeste do estado de São Paulo. Sociedade \& Natureza, Uberlândia, v. 23, n. 1, p. 7-22, abr. 2011.

BAÊTA, H. E. Contribuição da deposição úmida (chuva e neblina) nas relações hídricas e nutricionais de fisionomias de campos ferruginosos na Serra da Brígida, Ouro Preto, MG. 2012. 74 f. Dissertação (Mestrado em Ecologia de 
Biomas Tropicais) - Instituto de Ciências Exatas e Biológicas, Universidade Federal de Ouro Preto, Ouro Preto, 2012.

BENDIX, J.; FABIAN, P.; ROLLENBECK, R. In: INTERNATIONAL CONFERENCE OF FOG, FOG COLLECTION AND DEW, 3., 2004, Cape Town. Proceedings...Cape Town: University of Pretoria, 2004. 4 p.

BRUIJNZEEL, L. A. Hydrology of tropical montane cloud forests: a reassessment. Land Use and Water Research, Newcastle, v. 1, p.1.1-1.18, 2001.

BRUIJNZEEL, L. A.; HAMILTON, L. S. Tiempo decisivo para las selvas de neblina. Paris: UNESCO: International Hydrological Programme, 2001. 41 p. (IHP Programa Trópicos Humedos Serie no 13).

BRUIJNZEEL, L. A.; EUGSTER, W.; BURKARD, R. Fog as hydrologic input. In: ANDERSON, M. G. (Ed.). Encyclopedia of hydrological sciences. New Jersey: John Wiley \& Sons, 2005. cap. 38, p. 559-582.

BUBB, P; MAY, I. A.; MILES, L.; SAYER, J. Cloud forest agenda. Cambridge: PNUMA-CMVC, 2004. $32 \mathrm{p}$.

CERECEDA, P.; OSSES, P.; LARRAIN, H.; FARIAS, M.; LAGOS, M.; PINTO, R.; SCHEMENAUER, R. S. Advective, orographic and radiation fog in the Tarapacá region, Chile. Atmospheric Research, Amsterdam, v. 64, n. 1-4, p. 261-271, Sept.-Oct. 2002.

CICCO, V. Análise de séries temporais hidrológicas em microbacia com cobertura vegetal natural de Mata Atlântica, Cunha, SP. 2004. 124 f. Dissertação (Mestrado em Geografia Física) - Departamento de Geografia, Faculdade de Filosofia, Letras e Ciências Humanas, Universidade de São Paulo, São Paulo, 2004.

ESTRELA, M. J.; VALIENTE, J. A.; CORELL, D.; MILLÁN, M. M. Fog collection in the western Mediterranean Basin (Valencia region, Spain). Atmospheric Research, Amsterdam, v. 87, n. 3-4, p. 324-337, Mar. 2008.

EUGSTER, W. Fog research. Die Erde, Berlin, v. 139, n. 1-2, p. 1-10, 2008.

FISCHER, D.; STILL, C. J. Evaluating patterns of fog water deposition and isotopic composition on the California Channel Islands. Santa Barbara: University of California, 2007, 43 p. (Paper 2788).

FRUMAU, K. F. A.; BURKARD, R.; SCHIMID, S; BRUIJNZEEL, L. A ; TOBÓN, C; CALVO-ALVARADO, J. C. A comparison of the performance of three types of passive fog guages under conditions of wind-driven fog and precipitation. Hydrological Processes, Malden, v. 25, n. 3, p. 374-383, Jan. 2011.

FURIAN, S. M.; PFEIFER, R. M. Levantamento de reconhecimento do meio físico do Núcleo Cunha, SP. Boletim Técnico do Instituto Florestal, São Paulo, v. 40, n. 2, p. 183-193, dez. 1986.

FURIAN, S M. Estudo geomorfológico do escoamento superficial pluvial em parcelas experimentais no Parque Estadual da Serra do Mar - Núcleo Cunha / SP: um esboço metodológico. 1987. 187 f. Dissertação (Mestrado em Geografia Física) - Departamento de Geografia, Faculdade de Filosofia, Letras e Ciências Humanas, Universidade de São Paulo, São Paulo, 1987. 
GARCIA, A. L.; ARANGO, M. C. Evaluation and quantification of the hydrological potential of the fog at the Ipuana's hill, Guajira desert, Colombia, South America. In: INTERNATIONAL CONFERENCE OF FOG, FOG COLLECTION AND DEW, 3., 2004, Cape Town. Proceedings... Cape Town: University of Pretoria, 2004. 4 p.

GOODMAN, J. The collection of fog drip. Water Resources Research, Malden, v. 21, n. 3, p. 392-394, Mar. 1985.

HAFKENSCHEID, R. L. L. J. Hydrology and biogeochemistry of tropical montane rain forests of contrasting stature in the Blue Mountains, Jamaica. 2000. $315 \mathrm{f}$. Ph.D. thesis - Vrije Universiteit, Amsterdam, 2000.

HIATT, C.; FERNANDEZ, D.; POTTER, C. Measurements of fog water deposition on the California Central Coast. Atmospheric and Climate Sciences, v. 2, p. 525531, 2012.

HOLWERDA, F.; BRUIJNZEEL, L. A.; MUÑOZ-VILLERS, L. E.; EQUIHUA, M.; ASBJORNSEN, $\mathrm{H}$. Rainfall and cloud water interception in mature and secondary lower montane cloud forests of central Veracruz, Mexico. Journal of Hydrology, Amsterdam, v. 384, n. 1-2, p. 84-96, 2010.

HOLWERDA, F.; BRUIJNZEEL, L. A.; SCATENA, F. N. Comparison of passive fog gauges for determining fog duration and fog interception by a Puerto Rican elfin cloud forest. Hydrological Processes, Malden, v. 25, n. 3, p. 367-373, 2010.

INSTITUTO BRASILEIRO DE GEOGRAFIA E ESTATÍSTICA. Manual técnico da vegetação brasileira. 2. ed. Rio de Janeiro: IBGE, 2012. 271 p. (Série Manuais Técnicos em Geociências, nº 1).

INTERNATIONAL DEVELOPMENT RESEARCH CENTRE. Fogwater collection system: a new technology of simple application and large economic and social impact. Ottawa: International Development Research Centre, 1993, 17 p.

JUVIK, J. O.; NULLET, D. Comments on "a proposed standard fog collector for use in high-elevation regions". Journal of Applied Meteorology, Boston, v. 34, n. 9, p. 2108-2110, Sept. 1995.

KLEMM, O. et al. Fog as a fresh-water resource: overview and perspectives. Ambio, v. 41, 221-234, 2012.

LIU, W. J.; WANG, P. Y; JIN, T. L.; PENG, J. L; WEN, Y. L. The importance of radiation fog in the tropical seasonal rain Forest of Xishuangbanna, South-West China. Hydrology Research, London, v. 39, n. 1, p. 79-87, 2008.

LOUW, C.; VAN HEERDEN, J.; OLIVIER, J. The South African fog-water collection experiment: meteorological features associated with water collection along the eastern escarpment of South Africa. Water SA, Gezina, v. 24, n. 4, p. 269-280, 1998.

MARZOL, M. V. Fog water collection in a rural park in the Canary Islands (Spain). Atmospheric Research, Amsterdam, v. 64, n. 1-4, p. 239-250, Sept.Oct. 2002.

MARZOL, M. V.; SANTOS, G. G. Time variations of fog during summer in the Canary Islands. In: INTERNATIONAL CONFERENCE OF FOG, FOG COLLECTION AND DEW, 3., 2004, Cape Town. Proceedings...Cape Town: University of Pretoria, 2004. 4 p. 
MARZOL, M. V.; MEGÍA, J. L. S. Fog water harvesting in Ifni, Morocco. An assessment of potential and demand. Die Erde, v. 139, n. 1-2, p. 97-119, 2008.

MARZOL, M. V.; SANCHES, J. L.; YANES, A. Meteorological patterns and fog water collection in Morocco and the Canary Islands. Erdkunde, v. 65, n. 3 p. 291-303, 2011.

MCJANNET, D.; WALLACE, J. S. Methodology for estimating cloud interception inputs to tropical rainforest. Black Mountain: CSIRO, 2006. 14 p. (CSIRO Land and Water Science Report 6/6).

MCJANNET, D; WALLACE, J. S.; FITCH, P.; REDDELL, P. Water balance measurements in Australia's wet tropics: sites, methods and results. Black Mountain: CSIRO, 2006. 30 p. (CSIRO Land and Water Science Report 19/6).

MILETA, M. Fog water collection on the Croatian mountain. Balwois, Republic of Macedonia, v. 27, 3 p., May 2008.

MOLINA, J. M.; ESCOBAR, C.M. Fog collection variability in the Andean Mountain range of southern Colombia. Die Erde, v. 139, n. 1-2, p. 127-140, 2008.

MONTECINOS, S.; CERECEDA, P.; RIVERA, D. Fog collection and its relationship with local meteorological variables in a semiarid zone in Chile. Atmósfera, v. 31, n. 2, p. 143-153, 2018.

MONTEIRO, C. A. F. A dinâmica climática e as chuvas no Estado de São Paulo: estudo geográfico sob a forma de atlas. 1973. 129 f. Tese (Doutorado) Instituto de Geografia, Universidade de São Paulo, São Paulo, 1973.

MOUSAVI-BAYGI, M. The implementation of fog water collection systems in Northeast of Iran. International Journal of Pure and Applied Physics, Alexandria, v. 4, n. 1, p. 13-21, 2008.

OLIVIER, J. Fog-water harvesting along the West Coast of South Africa: a feasibility study. Water SA, v. 28, n. 4, p. 349-360, 2002.

OLIVIER, J.; RAUTENBACH, C. J. The implementation of fog water collection systems in South Africa. Atmospheric Research, Amsterdam, v. 64, n. 1-4, p. 227-238, Sept.-Oct. 2002.

OSSES, P; SCHEMENAUER, R. S.; LEIBBRAND, M. Fog collection evaluation in the Sadaa Governorate, Yemen. In: INTERNATIONAL CONFERENCE OF FOG, FOG COLLECTION AND DEW, 3., 2004, Cape Town, Proceedings...Cape Town: University of Pretoria, 2004. 4 p.

PRADA, S. N.; SILVA, M. O. Fog precipitation on the Island of Madeira (Portugal). Environmental Geology, Berlin, v. 41, n. 3-4, p. 384-389, 2001.

PRADA, S. N.; SILVA, M. O.; FIGUEIRA, C.; SEQUEIRA, M. M.; PONTES, A. Fog water collection in Madeira Island (Portugal). In: INTERNATIONAL CONFERENCE OF FOG, FOG COLLECTION AND DEW, 3., 2004, Cape Town. Proceedings...Cape Town: University of Pretoria, 2004. 4 p.

PRADA, S. SEQUEIRA, M. M.; FIGUEIRA, C.; VASCONCELOS, R. Cloud water interception in the high altitude tree heath forest (Erica arborea L.) of Paul da Serra Massif (Madeira, Portugal). Hydrological Processes, Malden, v. 26, n. 2, p. 202-212, 2012. 
SCHEMENAUER, R. S.; CERECEDA, P. A proposed standard fog collector for use in high-elevation regions. Journal of Applied Meteorology, Boston, v. 33, n. 11, p. 1313- 1322, Nov. 1994a.

SCHEMENAUER, R. S.; CERECEDA, P. Fog collection's role in water planning for developing countries. Natural Resources Forum, v. 18, n. 2, p. 91-100, 1994b.

SCHEMENAUER, R. S.; CERECEDA, P. Reply to comments by Juvik and Nullet (1995). Journal of Applied Meteorology, Boston, v. 34, n. 9, p. 2111-2112, Sept. 1995.

SCHEMENAUER, R. S.; JOE, P. I. The collection efficiency of a massive fog collector. Atmospheric Research, Amsterdam, v. 24, n. 1-4, p. 53-69, Dec. 1989.

SCHEMENAUER, R. S.; OSSES, P.; LEIBBRAND, M. Fog collection evaluation and operational projects in the Hajja Governorate, Yemen. In: INTERNATIONAL CONFERENCE OF FOG, FOG COLLECTION AND DEW, 3., 2004, Cape Town. Proceedings...Cape Town: University of Pretoria, 2004. 4 p.

SHANYENGANA, E. S.; HENSCHEL, J. R.; SEELY, M. K.; SANDERSON, R. D. Exploring fog as a supplementary water source in Namibia. Atmospheric Research, Amsterdam, v. 64, n. 1-4, p. 251-259. Sept.-Oct. 2002.

TANAKA, N. et al. Effects of fog on the water budget of a hill evergreen forest in Northern Thailand. In: ASIA PACIFIC ASSOCIATION OF HYDROLOGY AND WATER RESOURCES CONFERENCE, 3., 2006, Bangkok. Proceedings... Bangkok: APHW, 2006. 8 p.

WORLD METEOROLOGICAL ORGANIZATION. Measurement of precipitation. In: WORLD METEOROLOGICAL ORGANIZATION (Ed.). Guide to meteorological instruments and methods of observation. Geneva: Chalrperson Publications Board, 2008, cap. 6, p. 1.6/1-1.6/18.

VALIENTE, J. A.; ESTRELA, M. J.; CORELL, D. A fog collection network in the Valencia region (Western Mediterranean Basin). In: INTERNATIONAL CONFERENCE OF FOG, FOG COLLECTION AND DEW, 3., 2004, Cape Town. Proceedings...Cape Town: University of Pretoria, 2004. 4 p.

VALIENTE, J. A.; CORELL, D.; FUENTES, D; VALDECANTOS, A. Fog water collection and reforestation at mountain locations in a western Mediterranean basin region. INTERNATIONAL CONFERENCE ON FOG, FOG COLLECTION AND DEW, 5., 2010, Münster, Germany. Proceedings..., 2010, 5 p.

VALIENTE, J. A.; ESTRELA, M. J.; CORELL, D.; FUENTES, D; VALDECANTOS; BAEZA, M. Fog water collection and reforestation at a mountain location in a western Mediterranean basin region: air-mass origins and synoptic analysis. Erdkunde, v. 65, n. 3 p. 277-290, 2011. 